\title{
PARAMETRIC STUDY OF NEAR-WAKE STRUCTURE OF SPHERICAL AND CYLINDRICAL BODIES IN THE LABORATORY
}

\author{
W. A. ORAN and N. H. STONE \\ Space Sciences Laboratory, Marshall Space Flight Center, Huntsville, AL., U.S.A. \\ and \\ URI SAMIR \\ Space Physics Research Laboratory, University of Michigan at Ann Arbor and \\ Department of Enviromental Sciences, Tel-Aviv University, Israel
}

(Received 10 July 1973)

\begin{abstract}
Some aspects of the interaction between metal bodies and streaming rarefied plasmas were studied in a newly constructed Plasma Wind Tunnel as part of an attempt to investigate (via simulation) phenomena relevant to the spacceraft/spacc plasma interaction. Detailed ncarwake ion current profiles for both spherical and cylindrical bodies at different body potentials $\left(\phi_{S}\right)$ and at different plasma flow parameters are presented. Various features of the profiles can be correlated, at least qualitatively, with both plasma and body characteristics. For example, the width of the wake zone appears proportional to the Debye length $\left(\lambda_{p}\right)$ and depends on the potential of the target body although it appears to be relatively insensitive to the ratio $S=V_{\text {nlow }} /\left(2 k T_{e} / M_{+}\right)^{1 / 2}$. The amplitude of the ion current peak(s) also appears proportional to $\lambda_{D}$ while, for fixed $\phi_{S}$, the location of the peak is directly related to $S$ and possibly dependent upon body geometry. The general importance of body geometry is qualitatively demonstrated. In addition, a discussion of the relevance of the above studies to previous in situ data obtained from the Ariel I and Gemini/Agena missions is given.
\end{abstract}

\section{INTRODUCTION}

A renewed and growing interest in the problem of the spacecraft-ionospheric plasma interaction has resulted from possible experimental openings in future space missions such as the Shuttle Sortie Laboratories and perhaps the U.S.-U.S.S.R. Apollo-Soyuz Test Program. This interaction is a special case of the fundamental physical problem of characterizing the zone of disturbance around bodies of arbitrary geometry, size, and surface material in flowing rarefied plasmas. There is, also, a renewed interest in groundbased laboralory experiments which, although not precisely simulating those interactions, can greatly compliment in situ observations of the zone of disturbance of bodies in flowing space plasmas. Many such laboratory simulation experiments have been conducted in the last decade, ${ }^{(1-9)}$ but were not correlated with the available in situ data.

The majority of the in situ data relevant to studies of the wake of satellites were obtained from plasma probes onboard the Explorer VIII, Ariel I, and Explorer XXXI satellites. ${ }^{(10)}$ These data, however, yielded flux distributions only in the very near wake region. No study in space has yet been performed to measure systematically the transverse and axial structure of the entire zone of disturbance of bodies. This is due to the fact that most of the in situ data used comes from measurements taken by probes mounted on the surfaces of single-body spacecraft. It is clear that probes mounted on a multiple-boom satellite, although very useful, could not yield a comprehensive axial/transverse description of the entire zone of disturbance. Therefore, if a comprehensive study is sought, a multi-body spacecraft system will be required. In the past, one such attempt was made to perform this type of study using a two-body system; i.e. the Gemini/Agena mission. ${ }^{(11)}$ This unfortunately yielded relatively little data. 
Laboratory simulation studies can, with relative ease, (at least in principle) provide an overall study of the zone of disturbance by having a well defined plasma flow over a stationary body of known characteristics.

In this paper we present transverse ion current profiles taken in the near wake region of geometrically different objects. Two stainless steel bodies were used; an $8 \mathrm{~cm}$ dia sphere and an $8 \mathrm{~cm}$ by $8 \mathrm{~cm}$ circular cylinder orientated with its axis orthogonal to the direction of the plasma flow. The sphere presents a circular cross section to the beam while the cylinder has a square cross section.

Data were taken under the following plasma flow conditions: $6.9 \leq S \leq 12.8$ and $11 \cdot 7 \leq\left[R_{0} / \lambda_{D}\right] \leq 25 \cdot 0$, where $S=$ 'Mach number' $=V_{\text {tlow }} /\left(2 \mathrm{k} T_{e} / M_{+}\right)^{1 / 2}, \quad R_{0}=$ body radius, and $\lambda_{D}=$ Debye length.

The body radius for the sphere and the cylinder are the same, and the two cross sectional areas are almost equal ([area cylinder/area sphere] $=1 \cdot 27$ ). Therefore, significant differences in the ion profiles for the two bodies taken at the same plasma flow regime should result from different geometries.

\section{APPARATUS}

The experiments were conducted in an 0.6 by $1.5 \mathrm{~m}$ cylindrical plasma wind tunnel recently constructed at NASA/George C. Marshall Space Flight Center. A schematic diagram of the system is shown in Fig. 1. An electron bombardment ion source was used in conjunction with a hot emissive wire to produce the flowing plasma. Argon was used as the working gas, and the cylindrical tank was evacuated with an $\mathrm{LN}_{2}$ baffled oil diffusion pump. The various flow parameters obtained in this system are given in Table 1 and are compared with nominal values typically encountered by ionospheric satellites. The ion beam energy was measured with a multi-grid retarding potential analyzer. The electron temperature and density were measured with a cylindrical Langmuir probe (dia of $0.1 \mathrm{~cm}$ and length of $1.5 \mathrm{~cm}$ ) in the conventional manner, but the point of inflection was also checked as to its location using the (i-v) first derivative.

The test bodies, which consisted of the cylinder and sphere, were placed $60 \mathrm{~cm}$ from the ion engine orifice, and transverse ion profiles were taken at various points along the wake axis with a shielded Faraday cup. The cylinder was orientated so that its axis was orthogonal to both the flow and the plane formed by the ion profiles.

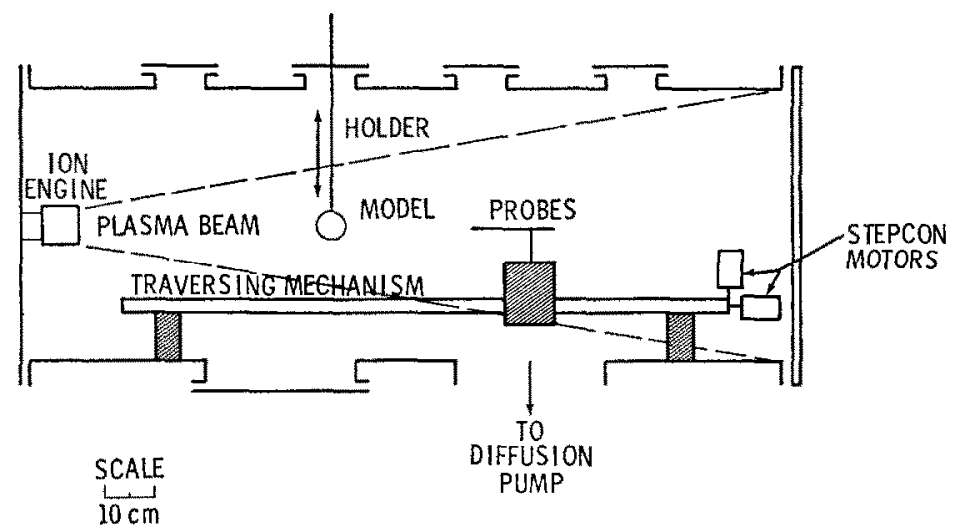

Fig. 1. A SCHEMATIC DRAWING OF the PLASMa WIND tUNNEL FACILITY. 
TABLE 1. SOME NOMINAL PlaSMa Flow PARAMETERS OF THE IONOSPHERE AND THE VALUES OF THE SAME PARAMETERS OBTAINED IN THE MSFC SIMULATION FACILITY

\begin{tabular}{|c|c|c|}
\hline Parameter & Ionospheric value & Simulation value \\
\hline $\begin{array}{l}\text { Neutral density } n \\
\text { Electron/ion density } n_{e,+} \\
\text { Electron temperature } T_{e} \\
\text { Ion temperature } T_{i}\end{array}$ & $\begin{array}{l}10^{11}-10^{18} / \mathrm{m}^{3} \\
10^{10}-10^{12} / \mathrm{m}^{3} \\
500-5000^{\circ} \mathrm{K} \\
500-5000^{\circ} \mathrm{K}\end{array}$ & $\begin{array}{l}\sim 3 \times 10^{19} / \mathrm{m}^{3} \\
10^{11}-10^{13} / \mathrm{m}^{3} \\
500-3000^{\circ} \mathrm{K} \\
\sigma 300^{\circ} \mathrm{K}^{*}\end{array}$ \\
\hline Electron Debye length $\lambda_{D}=\left(\frac{T_{e}}{4 \prod n_{l} l^{2}}\right)^{1 / 2}$ & $0.005-0.06 \mathrm{~m}$ & $0.001-0.01 \mathrm{~m}$ \\
\hline $\begin{array}{l}R_{0} / \lambda_{D} \\
\text { Ion mass } M_{+} \text {(amu) } \\
\text { Satellite velocity } V_{0}=V_{\text {rlow }}\end{array}$ & $\begin{array}{l}0.1 \text { to } 100 \\
1 \text { to } 30 \\
\sim 7 \mathrm{~km} / \mathrm{sec}\end{array}$ & $\begin{array}{l}1 \text { to } 50 \\
2 \text { to } 40 \\
\geq 5 \mathrm{~km} / \mathrm{sec}\end{array}$ \\
\hline Mach number $S=\frac{V_{0}}{\left(\frac{2 k T_{s}}{M_{+}}\right)^{1 / 2}}$ & 1 to 10 & $\geqslant 5$ \\
\hline
\end{tabular}

$l=$ electron charge

$k=$ Boltzman constant

$\phi_{s}=$ body potential relative to plasma potential

* Estimated value only.

\section{SOME RESULTS AND DISCUSSION}

Figures 2 and 3 show in matrix representation the transverse ion current profiles taken at different locations $\left[r / R_{0}\right]$ downstream in the wake zone for different body potentials and under different plasma flow conditions given in Table 2 . Figure 2 shows the profiles for the cylindrical body, and Fig. 3 shows the profiles for the spherical body. The presentation in Figs. 2 and 3 is such that each case refers to transverse measurements taken at different distances downstream for a set of fixed body potentials. If read horizontally, these transverse/axial profiles are similar to those presented by Stone et al. ${ }^{(8)}$ The ion source produces a diverging beam with the ion density decreasing by approx 50 per cent from $\left[r / R_{0}\right]=0$ to $\left[r / R_{0}\right]=8.5$ where $r$ is the axial distance to the body center and $R_{0}=4 \mathrm{~cm}$. Therefore, two values of $\lambda_{D}$ and the average $\bar{\lambda}_{D}$ are given for each case in Table 2.

\section{Basic structure in the wake region}

There is a void of ion flux in the center of the profiles taken in the very near wake region for both bodies. This void is flanked on either side by a plateau-like region where the ion current is a fraction of the ambient level. The plateau structure appears to originate from the deflection of the streaming ions by the body potential. The transverse ion profiles at $r / R_{0}=1.25$ are very similar for the sphere and cylinder under the same plasma flow conditions. There is still some similarity between equivalent spherical and cylindrical cases at $r / R_{0}=2$, although a peak structure is starting in some of the spherical cases. We find that for all values of $\phi_{S}$ given for the cylindrical body in Fig. 2, no peak in the ion current is seen until $\left(r / R_{0}\right)=3 \cdot 5$, and even then only for the more negative potentials. While for the cases given in Fig. 3 (sphere) some peak structure is seen already at $\left(r / R_{0}\right)=2$ for body potentials of $-2 \cdot 1 \mathrm{~V}$ and $-4 \cdot 1 \mathrm{~V}$.

For the cylindrical cases at distances larger than $\left(r / R_{0}\right)=3 \cdot 5$, a multiple peak structure is observed as opposed to a narrow single peak in the spherical cases (see Fig. 4). In no instance are the heights of the cylindrical body peaks significantly above ambient, while in some cases the heights of the spherical body peaks are approximately fifteen times the 

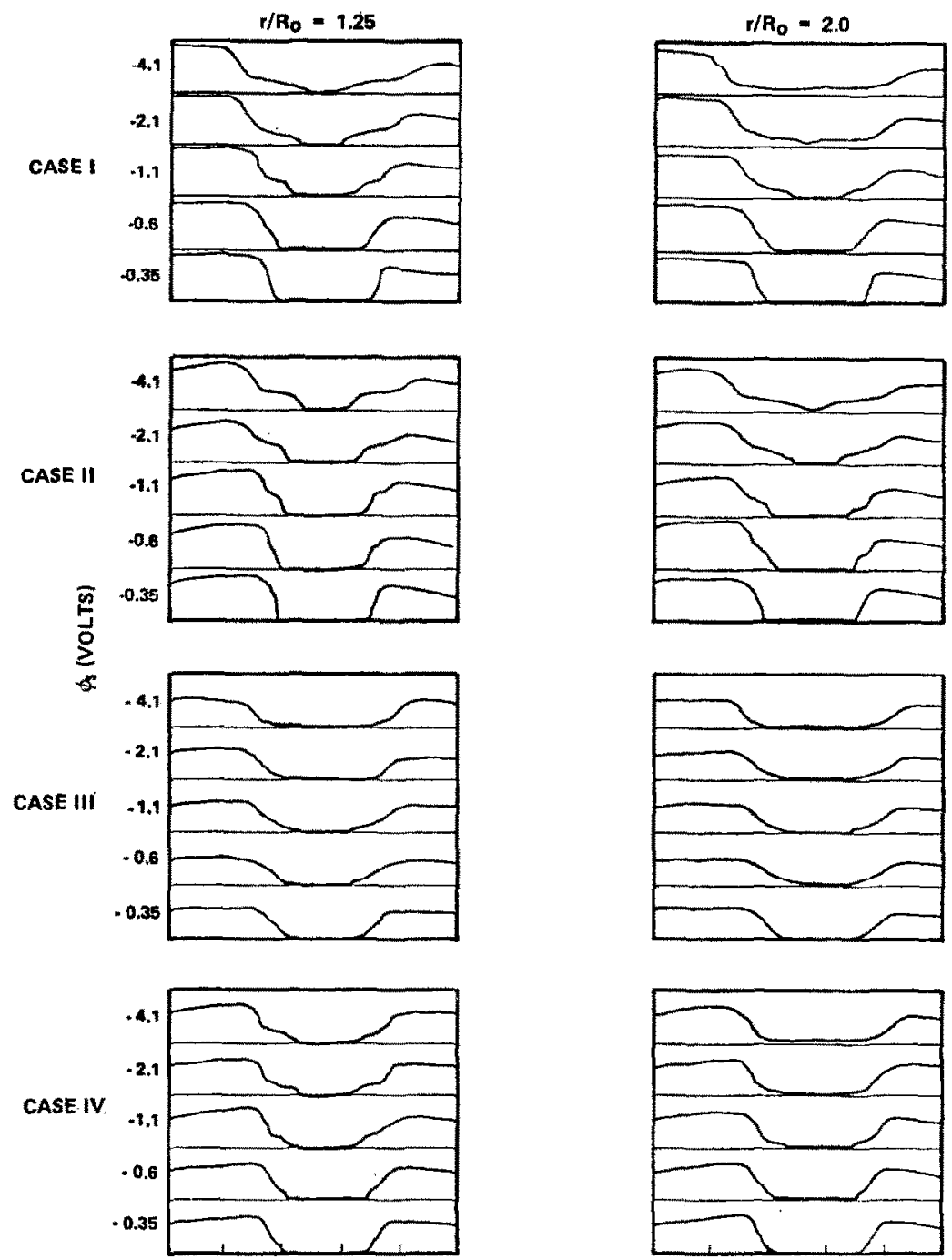

FIG. 2. MATRIX OF TRANSVERSE ION CURRENT PROFILES FOR THE CYLINDRICAL BODY FOR DIFFERENT BODY POTENTIALS $\phi_{S}$ AND DOWNSTREAM DISTANCES $r / R_{0}$ FOR THE CASES GIVEN IN TABLE 2.

ambient current (see Table 3 ). The peaks are relatively narrow for both cylindrical and spherical cases having a width of $0 \cdot 1-0.5 R_{0}$ depending on the plasma parameters and the distance $\left[r / R_{0}\right]$. The diameter of the entrance aperture of the shielded Faraday cup, for comparison, was $\sim 0.08 R_{0}$.

\section{Transverse width of disturbed zone}

Figure 5 shows the width $\left(\omega / R_{0}\right)$ of the zone of disturbance as a function of the distance downstream $r / R_{0}$ for the cylindrical Case II. The widths were measured from the first points on the ion profile where the current starts to deviate from the ambient. 

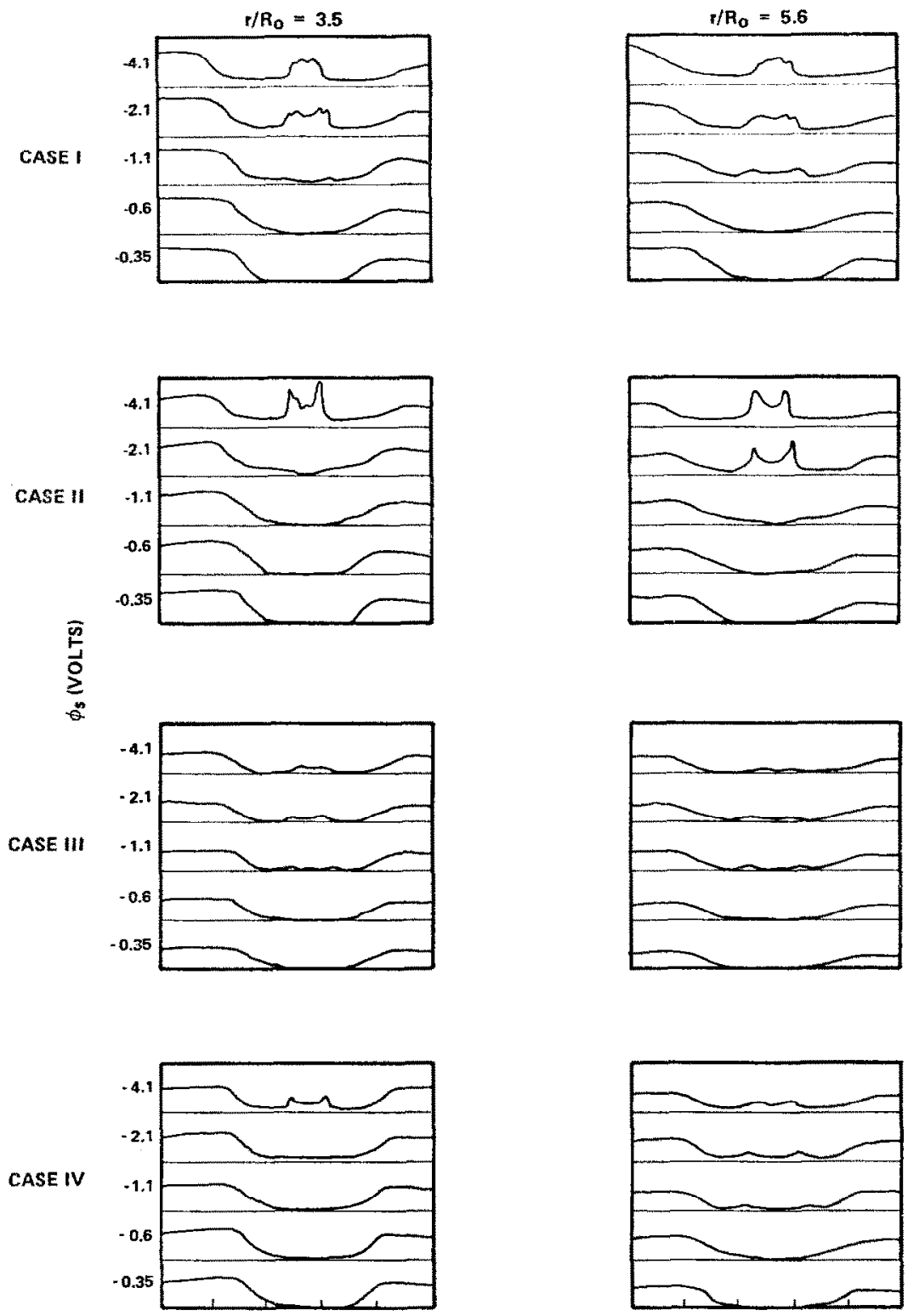

FIG. 2. (contd.)

Plots similar to Fig. 5 were produced for all cases, both spherical and cylindrical. Because of the errors introduced by the plasma beam divergence, an indication of the effective transverse width of the disturbed region caused by the body potential was determined by extrapolating the curves for $\phi_{S} \sim-0.4$ and $-4.1 \mathrm{~V}$ to $r / R_{0}=0$. The values of the widths measured for the cylindrical and spherical cases under equivalent plasma flow conditions were approximately equal. The measured values of the widths were relatively insensitive to the value of the Mach number in the range $6.9 \leq S \leq 12 \cdot 8$. 

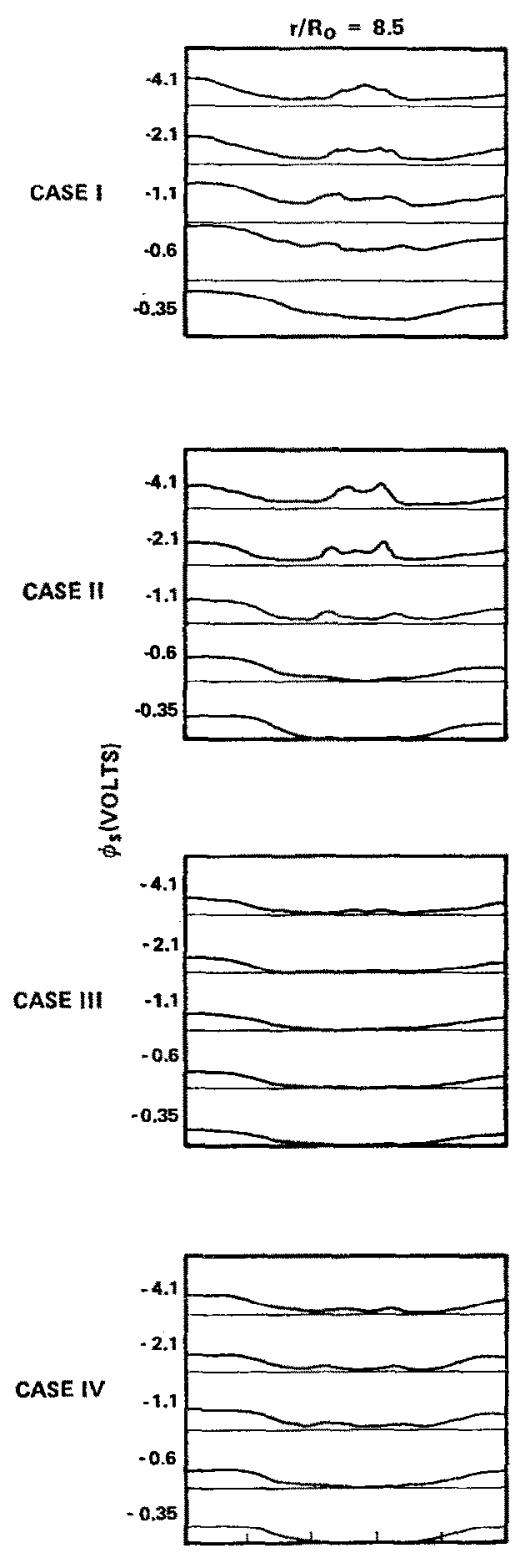

FIG. 2. (contd.)

However, the transverse width of the disturbed zone appeared proportional to $\bar{\lambda}_{D}$. Averaging the data for similar values of $\bar{\lambda}_{D}$, we find that for $\bar{\lambda}_{D} \approx 0.17 \mathrm{~cm}$, the zone of disturbance extends (in a transverse direction) $\approx 0.4 \mathrm{~cm}$ from the surface of a body with $\phi_{S} \approx-0.4 \mathrm{~V}$, and to $\approx 1.6 \mathrm{~cm}$ for $\phi_{S} \approx-4.1 \mathrm{~V}$. For $\bar{\lambda}_{D} \approx 0.32 \mathrm{~cm}$, the zone extends $\approx 0.8 \mathrm{~cm}$ for $\phi_{S} \approx-0.4 \mathrm{~V}$ and $\approx 2.9 \mathrm{~cm}$ for $\phi_{S} \approx-4.1 \mathrm{~V}$. The transverse width of the disturbed zone extended no more than $10 \bar{\lambda}_{D}$ even for the more negative body potentials used in the present set of experiments. 
CASE I
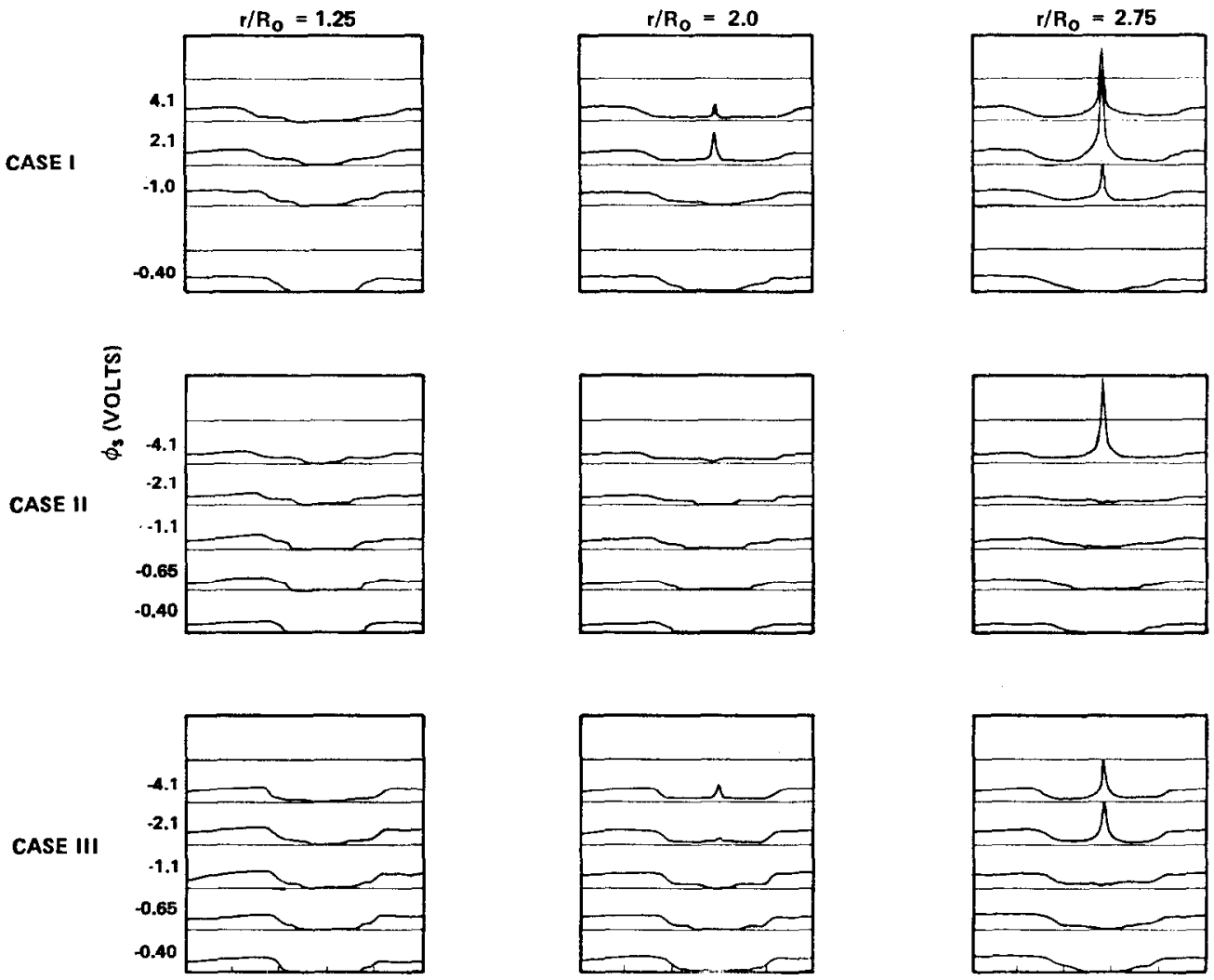

FIG. 3. MATRIX OF TRANSVERSE ION CURRENT PROFILES FOR THE SPHERICAL BODY FOR DIFFERENT BODY POTENTIALS $\phi_{S}$ AND DOWNSTREAM DISTANCES $r / R_{0}$ FOR THE CASES GIVEN IN TABLE 2 .

It should be noted that in Fig. 3 the general pattern is repeatable with the exception that 3 of the 40 ion peaks were not always reproducible; i.e. for Case III at $\phi_{s}=-1.1 \mathrm{~V}, r / R_{0}=3.5 ; \phi_{s}=-0.65 \mathrm{~V}, r / R_{0}=5.6 ;$ and $\phi_{s}=-0.40 \mathrm{~V}$, $r / R_{0}=8 \cdot 5$. This point requires further study.

\section{Effects due to the variation in Mach number}

For Cases I and II for the cylindrical body (Fig. 2), $\bar{\lambda}_{D}$ values are about the same but $S$ (Case II) $\approx 1.7 S$ (Case I). Comparing these cases for body-potential values of $\left|\phi_{S}\right|>1.1 \mathrm{~V}$ we find that noticeable (significant) peak structure starts at $\left(r / R_{0}\right)=3.5$ for Case I while a similar structure starts at $\left(r / R_{0}\right)=5.6$ and 8.5 for Case II. This implies that the peak in the ion current starts closer to the surface of the body for lower values of $S$. This same behavior is also seen for the spherical body (Fig. 3). Here again it will suffice to compare Cases I and II. This result is in general accord with Stone et al.,(12) where it was suggested that the maximum amplitude of the ion peak behind a sphere occurs at $\approx\left(S . R_{n}\right)$, indicating that the peak occurs closer to the body for lower Mach numbers.

4. Effects due to the variation in Debye length

Examination of Cases I-IV for the cylindrical body (see Table 2) where: $\bar{\lambda}_{D}$ (Case I) $/ \bar{\lambda}_{D}$ $\left(\right.$ Case III) $\approx \bar{\lambda}_{D}\left(\right.$ Case II) $/ \bar{\lambda}_{D}($ Case IV) $\approx 2$ (see Table 2$) ;[S$ (Case I) $\simeq S$ (Case III) $]$ and 

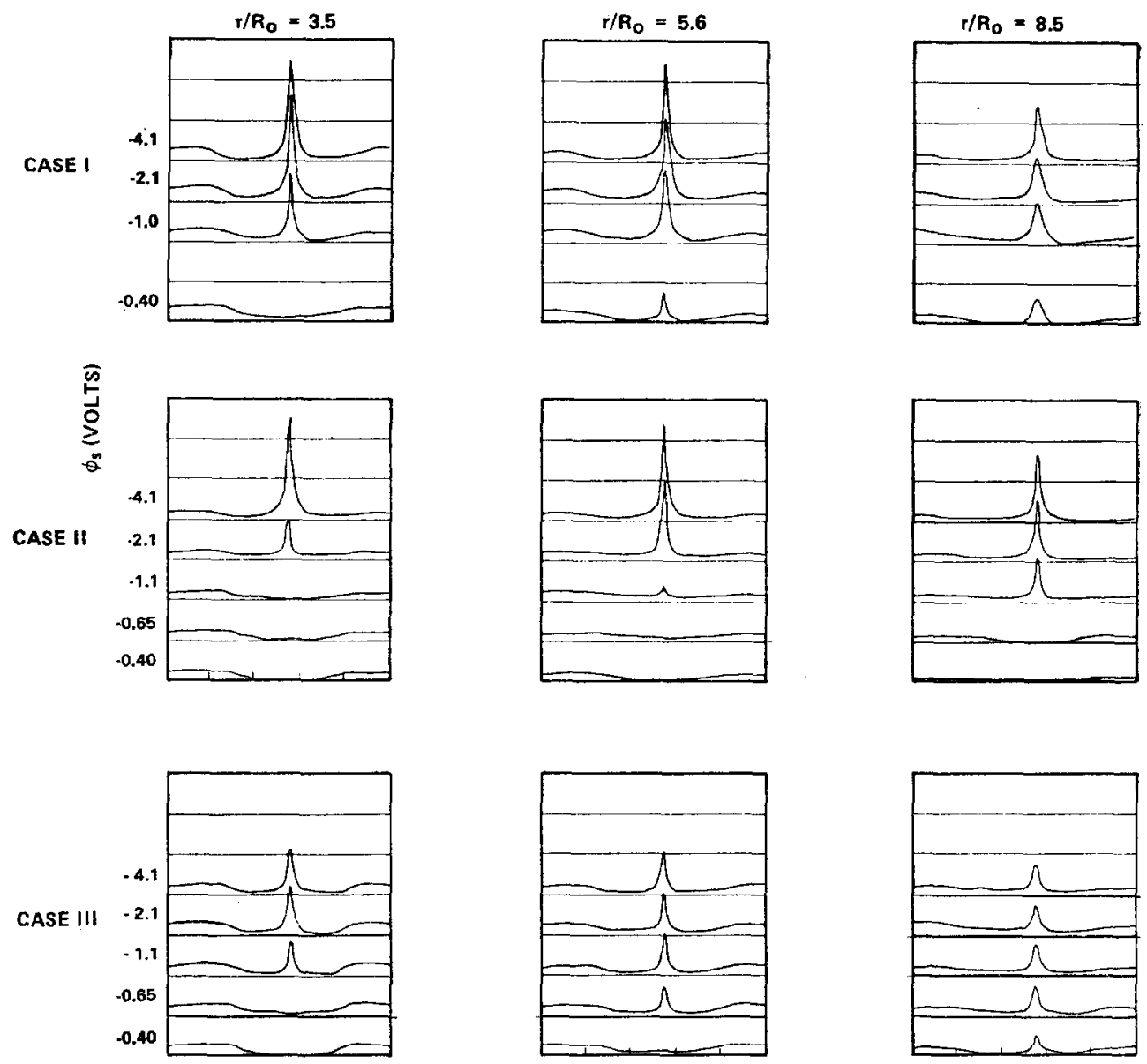

FIG. 3. (contd.)

$[S$ (Case II) $\simeq S$ (Case IV)] (to within 5 per cent) shows the amplitudes of the ion current peaks to be significantly higher (by $\sim$ a factor of 2 ) for higher values of $\bar{\lambda}_{D}$. A similar kind of behavior is also seen from Cases II and III for the spherical body (Fig. 3), where the same conditions of Debey ratio and Mach number apply. One can therefore conclude that for similar values of $\phi_{S}$ and $S$, the amplitude of the ion current peak in the near wake zones of both the cylindrical and spherical bodies depends directly on $\bar{\lambda}_{D}$. Note, however, that the above conclusion does not imply an equality in the amplitudes of the peaks for the cylindrical and the spherical bodies. As previously noted, the amplitude of the peak in the wake of the spherical body is larger than the corresponding amplitude of the ion current peak in the wake of the cylindrical body; i.e. on examining Case IV of the cylindrical body (Fig. 2) with Case III of the spherical body (Fig. 3) $I$ (peak)/I (ambient) for the cylinder is $\sim 1$ while this ratio is $\sim 15$ for the sphere. Since body potentials and plasma stream parameters are equivalent for both cases, this difference must be due to effects of geometrical shape.

The height of the plateau region observed in the very near wake (see Section 1.1) exhibited a similar dependence upon the Debye length. Namely, for the same Mach number, the height of this plateau relative to the ambient appears to be a factor of 2 greater for 
Table 2. Plasma flow parameters associated with the cases of the ion PROFILES GIVEN IN FIGS. 2 AND 3

\begin{tabular}{lcccc}
\hline \multirow{2}{*}{ Parameters } & \multicolumn{4}{c}{ Cylindrical runs (Fig. 2) } \\
\cline { 2 - 5 } & Case I & Case II & Case III & Case IV \\
\hline$T_{e}$ & $\sim 900^{\circ} \mathrm{K}$ & $\sim 1000^{\circ} \mathrm{K}$ & $\sim 900^{\circ} \mathrm{K}$ & $\sim 1100^{\circ} \mathrm{K}$ \\
$S$ & 7.4 & $12 \cdot 3$ & $7 \cdot 3$ & 12.0 \\
$\lambda_{D}$ at $\left(r / R_{0}=0\right)$ & $0.28 \mathrm{~cm}$ & $0.24 \mathrm{~cm}$ & $0.14 \mathrm{~cm}$ & $0.13 \mathrm{~cm}$ \\
$\lambda_{D}$ at $\left(r / R_{0}=8 \cdot 5\right)$ & $0.41 \mathrm{~cm}$ & $0.37 \mathrm{~cm}$ & $0.22 \mathrm{~cm}$ & $0.19 \mathrm{~cm}$ \\
$\bar{\lambda}_{D}$ & $0.34 \mathrm{~cm}$ & $0.31 \mathrm{~cm}$ & $0.18 \mathrm{~cm}$ & $0.16 \mathrm{~cm}$ \\
$R_{0} / \bar{\lambda}_{D}$ & 11.7 & 12.9 & 22.2 & 25.0
\end{tabular}

Spherical runs (Fig. 3)

\begin{tabular}{lccc}
\cline { 2 - 4 }$T_{*}$ & $\sim 900^{\circ} \mathrm{K}$ & $\sim 900^{\circ} \mathrm{K}$ & $\sim 900^{\circ} \mathrm{K}$ \\
$S$ & 6.9 & 12.6 & 12.8 \\
$\lambda_{D}$ at $\left(r / R_{0}=0\right)$ & $0.25 \mathrm{~cm}$ & $0.25 \mathrm{~cm}$ & $0.13 \mathrm{~cm}$ \\
$\lambda_{D}$ at $\left(r / R_{0}=8.5\right)$ & $0.34 \mathrm{~cm}$ & $0.41 \mathrm{~cm}$ & $0.19 \mathrm{~cm}$ \\
$\bar{\lambda}_{D}$ & $0.30 \mathrm{~cm}$ & $0.33 \mathrm{~cm}$ & $0.16 \mathrm{~cm}$ \\
$R_{0} \mid \bar{\lambda}_{D}$ & 13.4 & 12.1 & 25.0
\end{tabular}

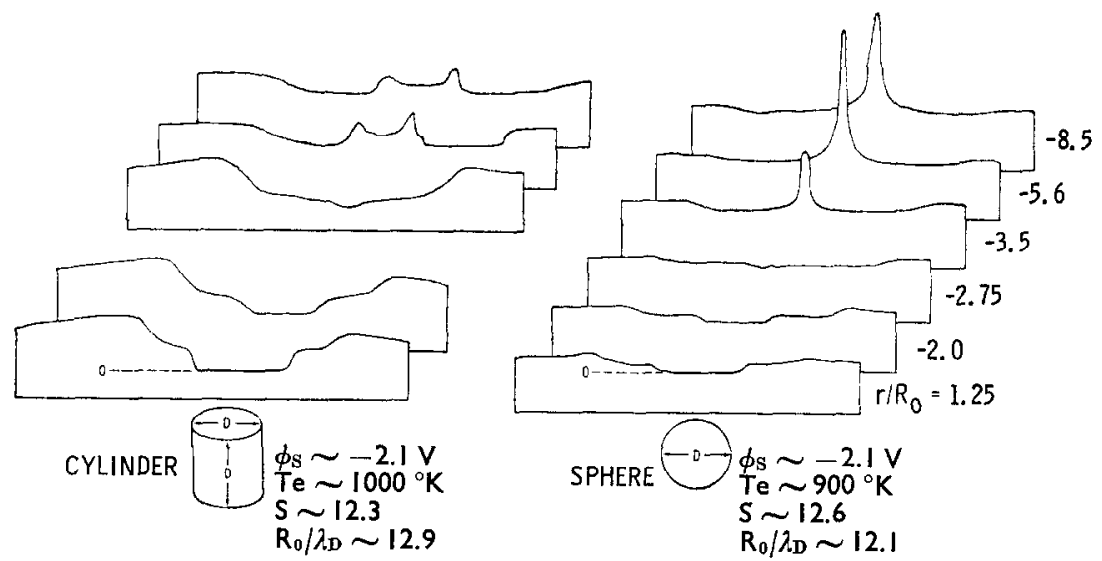

Fig. 4. COMParison OF THE NEAR-WAKe STRUCTURE OF A CYLINDER AND A SPHERE OF THE SAME DIAMETER AND SIMILAR PLASMA PROPERTIES.

The figures in the foreground are not intended for scaling but to show their orientation with respect to the plasma flow.

the cases with the larger $\bar{\lambda}_{D}$ (the ratios of $\bar{\lambda}_{D} \sim 2$ ). This is true for both the spherical and cylindrical cascs.

Observing Cases II and III in Fig. 3, which have approximately the same Mach number, we find that for a given $\phi_{S}$ the single peak structure begins nearer to the body than for the case of the smaller $\bar{\lambda}_{D}$. It could be argued that in Case III, which has the smaller $\bar{\lambda}_{D}$, the electric field at the sphere surface is greater and hence, with the greater deflective force, the ions will be focused at a closer axial distance to the sphere.

It is of interest to note that for relatively less negative potentials (e.g. $\phi_{S}=-0.4 \mathrm{~V}$ ) a peak is obtained for the spherical body (see Case I and Fig. 3). No such structure is seen for the cylindrical body (Fig. 2), for a similar potential value (i.e. $\phi_{S}=-0.35 \mathrm{~V}$ ). This difference between the structure in the wake of the sphere and cylinder is most likely due to geometry/size effects. However, this conclusion is based (solely) on the fact that 
TABle 3. Normalized values OF THE ION CURRENT PEAKS WHICH ARE FOUND AT THE CENTER OF THE WAKE PROFILES FOR THE SPHERICAL CASES GIVEN IN FIG. 3*

\begin{tabular}{clrrc}
\hline$\phi_{s}$ & $r / R_{0}$ & Case I & Case II & Case III \\
\hline$-4.1 \mathrm{~V}$ & 2.75 & 7 & 13 & 5 \\
& 5.6 & 7 & 21 & 7 \\
$-2.1 \mathrm{~V}$ & 8.5 & 11 & 17 & 6 \\
& 2.75 & 8 & 1 & 5 \\
$\sim-1.1 \mathrm{~V}$ & 5.6 & 10 & 15 & 6 \\
& 8.5 & 9 & 15 & 6 \\
$-0.65 \mathrm{~V}$ & 2.75 & 3 & 0 & 0 \\
& 5.6 & 10 & 3 & 5 \\
$-0.40 \mathrm{~V}$ & 8.6 & 7 & 10 & 5 \\
& 5.5 & - & 0 & 0 \\
& 8.5 & 3 & 0 & 1 \\
\hline
\end{tabular}

* The value of the ion current at the peak is normalized with respect to ambient.

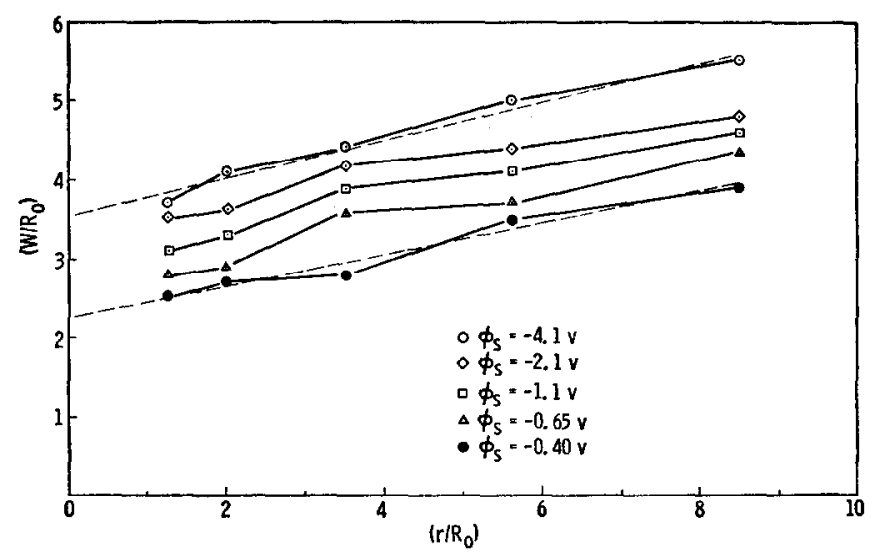

FIg. 5. TOTAL TRANSVERSE WIDTHS OF THE ZONE OF DISTURBANCE $\omega / R_{0}$ CREATED BY THE CYLINDRICAL BODY IN THE PLASMA FLOW CONDITIONS GIVEN IN CASE II AS A FUNCTION OF DISTANCE DOWNSTREAM $\left[r / R_{0}\right]$.

The dotted line is an extrapolation of the width of disturbance to the line $r / R_{0}=0$.

the plasma parameters of Case I for the cylinder are close to Case I for the sphere, and requires further study. A general illustration of the geometry/size effect is provided in Fig. 4.

5. A qualitative comparison between the results of the present study and those from in situ observations

The wake structure of the spherical body (see Figs. 3 and 4) resembles to some extent results obtained from the Ariel I satellite. ${ }^{(13)}$ There, an enhancement in electron current $\left(I_{e}\right)$ was observed at $r \sim 5 R_{0}$ with a boom mounted probe ( $S$ was approx 4 ). $R_{0}$ is the effective satellite radius. The amplitude of this enhanced $I_{e}$ was less than the value of the corresponding ambient $I_{e}$.

A similar $I_{e}$ enhancement was observed in the wake of a spherical ion-probe mounted on the spin axis of the Ariel I satellite and acting as a wake generator (target body). The 
boom mounted electron probe was also used in these measurements. However, in this casc, the obscrvation was obtained at a distance of $33 R_{P}$ downstream, where $R_{P}$ is the ion probe radius. Therefore, from the Ariel I data we observe enhancements in the electron currents behind 'target bodies' at $\sim S . R_{0}$ and at a distance $\gg S . R_{P}$. This compares favorably with our laboratory data in which the peak of the ion current enhancement was observed at a distance $\sim S . R$ and the enhancement of the ion current was observed to extend to distances $\gg S . R$ (see Stone et al. ${ }^{(12)}$ ).

We should state that the potential of the ion-probe was significantly more negative (by a factor of $\approx 7$ as shown in Henderson and Samir ${ }^{(13)}$ ) than the potential of the main body of the satellite $\left(\phi_{S} \approx-1 \mathrm{~V}\right)$ with respect to the environmental plasma and the ratio $\left[R_{P} / \lambda_{D}\right]=0.17\left[R_{0} / \lambda_{D}\right]$. The influence of body potential on the location of the peak amplitude in ion current was discussed in Lederman et al. ${ }^{(8)}$ and Stone et al. ${ }^{(12)}$ based on laboratory work. In addition, Fig. 5, which indicates how the 'effective cross-section' of a body is dependent upon $\phi_{S}$ for a limited range of flow parameters, may be helpful in explaining the approximately equal width of the disturbed zone behind the ion probe and Ariel I (see Henderson and Samir ${ }^{(13)}$ ).

One axial profile for electron and ion currents in the wake of the Gemini 10 manned capsule (measured by probes mounted on the Agena vehicle) was presented in Troy $\boldsymbol{e} t$ al. ${ }^{(11)}$ It is unfortunate that the above profile is, at present, the only one known to the authors of this paper to exist in the open literature. However, if we are to use the above finding, then there is a disagreement between the Gemini/Agena and the laboratory/Ariel I results regarding electron and/or ion current enhancement on the wake's axis (downstream). No such enhancement was reported for the Gemini. It is difficult to point out a reason why such a discrepancy should exist. However, based on our laboratory work, we may perhaps speculate and suggest that the narrowness of the enhanced current peaks (on the wake's axis) on one hand and the uncertainties as to the precise location of the Agena relative to the Gemini axis on the other hand may be contributing factors for not detecting enhancements. A peak structure has usually been observed in the downstream wake zone of bodies in laboratory 'simulation' systems. The most relevant profiles were those involving a model of an Apollo vehicle presented in Stone et al. ${ }^{(12)}$ in which a narrow peak was observed in the near wake zone. We should also bear in mind that the ionospheric properties and hence the relevant plasma parameters for both the Gemini and the Ariel I were not very different.

\section{SUMMARY}

We have investigated the near wake zone for a spherical and cylindrical body in a system which simulates to some extent the spacecraft/space plasma interaction. Data for both bodies were obtained under similar plasma flow conditions. A multi peak structure was observed in the wake of the cylindrical body in which the height of the peaks never rose significantly above ambient. A single peak whose amplitude could be as much as fifteen times ambient was observed downstream from the spherical body. The validity of the interpretation of the single vs multi-peak structure as a function of body geometry requires further study.

In the present study we were concerned with the overall structural differences seen in the wakes of spherical and cylindrical bodies in a limited range of plasma parameters of $6.9 \leq S \leq 12.8,11.7 \leq R_{0} / \bar{\lambda}_{D} \leq 25.0,-4.1 \mathrm{~V} \leq \phi_{S} \leq-0.35 \mathrm{~V}$. Basically, cases were examined where any two of the parameters $\phi_{S}, S$, and $\bar{\lambda}_{D}$ were approximately 
fixed and the third was varied. In this way, the relative peak amplitudes, the transverse width of the disturbed region, the location of the structure downstream, and the fine structure in the very near wake zone were found to depend upon the parameters $\phi_{S}, S$, and $\bar{\lambda}_{D}$ to various degrees. Therefore, $\phi_{S}, S, \bar{\lambda}_{D}$, and the geometrical shape of the bodies must all be considered as significant parameters in describing the zone of disturbance created by a body in a plasma flow field.

Some speculative comparisons were made between our laboratory wake data and that resulting from in situ measurements. Quantitative comparisons of this type involving more complex bodies in plasmas having a wider range of flow parameters will help to better understand the importance of various parameters in the spacecraft/space plasma interaction. Even qualitative studies as made in this paper are of practical interest in planning measurements for large spacecraft. Included in this group would be spacecraft such as the Space Shuttle and/or the vehicles involved in the Apollo Soyuz Test Program.

Acknowledgement- - One of the authors (U. S.) wishes to acknowledge the support under grants NGR 23-005. 320 and NGR 23-005-151.

\section{REFERENCES}

1. D. F. HALL, R. F. Kemp and J. M. Sellen, JR., ALAA J. 2 (6), 1032 (1964).

2. D. F. HALL, R. F. KEMP and J. M. SELLEN, JR., $A I A A J .3$ (8), 1490 (1965).

3. W. A. Clayden and C. V. HuRdLe, Rarefied Gas Dyn. 2 (4), 1717 (1966).

4. V. V. Skvortsov and L. V. NosACHEv, Kosmicheskie Issledovaniya 6 (2), 228 (UDC 533.9.07) (1968).

5. I. A. Bogashchenko, A. V. GUREvich, R. A. SAlimov and YU. I. EIDEL'MAN Soviet Phys. JETP 32 (5), 841 (1971).

6. S. D. Hester and A. A. Sontn, Physics Fluids 13 (3), 641 (1970).

7. S. D. Hester and A. A. SonIN, $A I A A$ J J. 8, 1090 (1970).

8. N. H. Stone, W. A. Oran and U. Samir, Planet. Space Sci. 20, 1787 (1972).

9. S. Lederman, M. H. Bloom and G. F. WidHonf, AIAA J. 7, 1421 (1969).

10. U. SAMIR, Israel J. Technol. 10, 179 (1972).

11. B. TroY, JR., D. MEDVED and U. SAMIR, J. astr. Sci. 18 (3), 173 (1970).

12. N. H. Stone, U. SAMIR and W. A. Oran, J. atmos. terr. Phys. 36, 253 (1974).

13. C. L. Henderson and U. SAMIR, Planet. Space Sci. 15, 1499 (1967). 